ÉGYPTE

monde arabe

\section{Égypte/Monde arabe}

4-5| 2001

L'Égypte dans le siècle, 1901-2000

\title{
Un emploi pour un diplôme
}

L'usure d'un accord tacite (1930-1990)

\section{Iman Farag}

\section{OpenEdition}

\section{Journals}

Édition électronique

URL : https://journals.openedition.org/ema/874

DOI : $10.4000 /$ ema. 874

ISSN : 2090-7273

Éditeur

CEDEJ - Centre d'études et de documentation économiques juridiques et sociales

Édition imprimée

Date de publication : 30 juin 2001

Pagination : 173-190

ISBN : 2-87027-963-9

ISSN : 1110-5097

Référence électronique

Iman Farag, "Un emploi pour un diplôme », Égypte/Monde arabe [En ligne], 4-5 | 2001, mis en ligne le 08 juillet 2008, consulté le 07 juillet 2022. URL : http://journals.openedition.org/ema/874 ; DOI :

https://doi.org/10.4000/ema.874

Ce document a été généré automatiquement le 7 juillet 2022.

Tous droits réservés 


\title{
Un emploi pour un diplôme
}

\author{
L'usure d'un accord tacite (1930-1990)
}

\section{Iman Farag}

« La propagation de l'instruction étant une condition essentielle du progrès et de la prospérité générale du pays et du bien-être individuel de ses habitants, son altesse feu Muhammad 'cAlî pacha ordonna la création des makatibs et des écoles en Égypte. Le

gouvernement égyptien ayant besoin à cette époque d'un grand nombre de personnes pouvant remplir les diverses fonctions tant civiles que militaires, fut forcé de recruter son personnel parmi les jeunes gens ayant terminé leurs études

dans ces écoles. C'est ce qui induisit le public à croire que le gouvernement était tenu d'employer

les jeunes gens sortant des écoles et de les entretenir, qu'ils fussent ou non aptes à remplir les fonctions à eux confiées ou bien même que le

gouvernement ait besoin ou non de leurs services. Or, la création des écoles remontant déjà à une époque ancienne et le nombre des hommes capables allant toujours croissant, le gouvernement se sent à présent libre de faire son choix parmi eux pour occuper les places vacantes dans les divers services de l'État. En conséquence, des règles générales ont été établies pour l'admission du personnel dans les divers services de l'État et les élèves des écoles ont été avisés à plusieurs reprises que le gouvernement n'était aucunement tenu de les employer en dehors de

ses besoins. Cependant, cette idée fausse et 


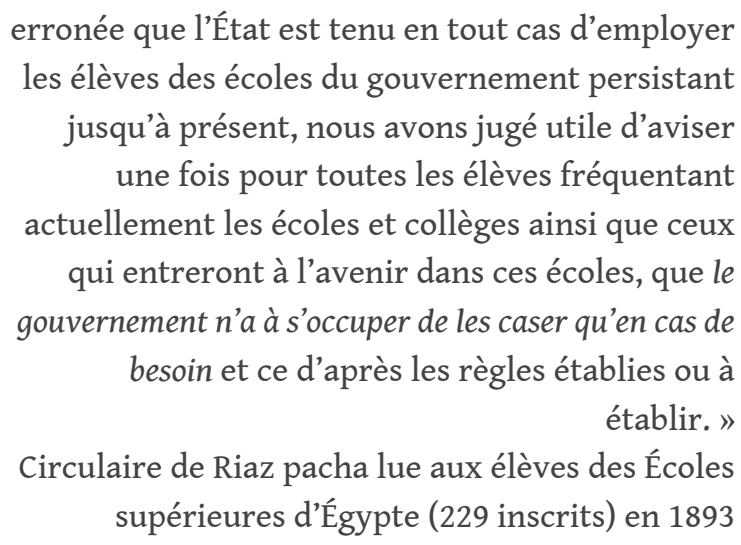

1 Force est de constater que l'avis a été maintes fois repris, puisque, selon la loi 43 de 1985, l'État n'embauche désormais les diplômés qu'en fonction de ses besoins. Il est ainsi possible de relever, sur un siècle, la répétition de situations qui mettent en lien et en scène éducation et emploi. Entre ce qui se présente comme une nécessité toute bureaucratique - les "besoins de l'État", en fait ce qu'il faut pour gérer et peut-être faire progresser la collectivité - et ce que fonde, à son insu quelquefois, cette représentation productrice d'attentes légitimes en termes de lien politique, la frontière est difficile à établir. Le tout permet d'esquisser quelques pistes de réflexion sur le destin et les fluctuations d'une obligation (morale) d'État qui n'était pas pour autant un droit.

2 Comparer, ne va pas sans risques, notamment quand il s'agit des différents états d'un même objet - le rapport politique entre emploi et éducation - à travers le temps. Et en ne retenant de surcroît pour tout principe de découpage que des "temps chauds", qui s'imposent avec une objectivité toute faite. En outre, les deux moments retenus sont l'Égypte républicaine et "néo-libérale" des années 1990 et l'Égypte "libérale" et monarchique des années 1930 : les comparer, c'est les séparer. La coupure en est-elle une ? Interroger ces deux moments est tout autant retour sur l'entre-deux : années 1960, Égypte nassérienne pour les initiés, ou encore "État social" dans un langage plus générique. Les deux histoires que l'on racontera ne sont pas homologues; celle des années 1990 met en scène les destins d'un groupe, les enseignants, alors que pour les années 1930, il s'agit de la question du chômage des diplômés et sa construction. Cela dit, des analogies sont possibles et les plus mineures et purement phénoménologiques en apparence, ne sont pas les plus triviales. On achèvera sur quelques interrogations.

Août 1998 - avril 1999 : des enseignants aux « élèves-maîtres »

3 La première histoire concerne les enseignants. Pourquoi ceux-là plus que d'autres ? Il s'agit du plus grand corps de l'État après l'armée, soit à peu près 1200000 dont environ 800000 enseignants; en termes d'opportunités d'emploi, ce sont les plus tributaires de la fonction publique puisque l'enseignement privé n'en absorbe qu'une part infime; ceux dont on parle le plus au titre de principaux responsables des dysfonctionnements du système d'enseignement ; ceux qui parlent le moins, puisque les plus modestes dans l'échelle de noblesse des professions universitaires qui les place bien loin des ingénieurs ou des médecins, et parce que toute prise de parole des enseignants se heurte au poids de cette machine à produire de la hiérarchie administrative qu'est l'appareil de l'éducation nationale. Leur organisation syndicale en est le calque. Avec les enseignants, on est bien au cœur de la reproduction du rapport emploi /éducation. Lequel est vu comme un dilemme par les décideurs du monde entier qui n'en 
retiennent qu'une seule des formulations, la plus hégémonique et « réaliste » il est vrai, qui consiste à agir sur la formation en fonction des données de l'emploi. Enfin, si l'Égypte des années 1990 n'a guère connu jusqu'ici ces mouvements de diplômés chômeurs - ou de chômeurs tout court - observés ailleurs, c'est bien à propos des enseignants que s'est posée de la manière la plus explicite la question des obligations de l'État vis-à-vis de ses diplômés.

4 C'est un micro-événement qui en fournira le prétexte. Le changement de mode de recrutement des enseignants - passage de l'embauche systématique au concours - a eu lieu en août 1999. L'événement n'aurait pas eu l'écho qui fut le sien s'il n'avait été accompagné d'un « effet de masse » fortuit : centralité valant sans doute pour équité, toutes les démarches de candidature devaient se faire au Caire et c'est donc plus de 275 000 candidats qui ont afflué de tous les coins d'Égypte vers la capitale, prenant d'assaut trains et micro-bus. Pour ou contre 50000 postes, autant dire que le " rassemblement " (Joseph, 1996, p. 107-122) - qui sans dégénérer a pourtant été fortement canalisé par les forces de sécurité - résumait à lui seul quelque 225000 déclinaisons virtuelles du « sens de la justice»; pour autant que celle-ci se définisse, par la négative, comme le sentiment d'injustice.

5 Ce n'est pas ce qu'ont relevé d'abord les observateurs. Les commentaires de la presse qui couvrait discrètement l'événement rendaient compte avant tout d'un étonnement certain. Que dans une économie libéralisée, et qu'au moment où est promu le modèle du self-made man, un tel nombre de candidats se bouscule aux portillons de postes aux salaires aussi peu prometteurs, cela semblait incompréhensible. Il faut savoir que le salaire moyen d'un enseignant (140 livres égyptiennes) place celui-ci légèrement audessus du seuil de pauvreté s'il est célibataire. Explication conjoncturelle, l'afflux de candidats aux postes d'enseignants s'expliquait par le temps de latence que nécessite l'ajustement des cycles : l'offre en diplômés excède les nouveaux besoins du marché ou encore les besoins d'un marché nouveau. Il y eut également des interprétations à prétention plus psychosociologique: on s'en prenait ici à la rationalité, sinon la mentalité, de ceux qui préféraient encore l'assurance d'un poste dans la fonction publique et le salaire qui va avec, au goût de l'aventure, avec ses aléas et promesses. L'argument porte plus large que la population des enseignants; c'est, de manière générale, toute recherche de tels postes qui serait témoin et de l'incompétence de ceux qui n'ont pas trouvé mieux, et de ce que serait devenue la fonction publique: une source de rente mesquine à laquelle ne répond de surcroit aucun « travail » (c'est en de telles occasions qu'on fait appel aux "productivités comparées », Égypte et Japon par exemple). Il était question enfin de cumul et d'usage de la fonction publique à des fins privées: les candidats aux postes d'enseignants ont déjà des emplois ou encore, s'ils recherchent ces postes, c'est uniquement pour pouvoir donner des cours particuliers, éminemment plus lucratifs que le salaire d'enseignant.

6 L'événement révélait la sociologie honteuse des acteurs. Les Facultés de Pédagogie dont sont issus la plupart des candidats sont bien le dernier échelon de la distinction universitaire : n'y accèdent que ceux qui, là aussi, n'ont pas trouvé mieux, puisque c'est dans les Facultés de Pédagogie - et sans aucun choix ni vocation de leur part - qu'un faible et implacable pourcentage au bac les a placés. Pis, il serait question non seulement de leurs ressources intellectuelles, mais de leurs ressources tout court : ceux contraints par la proximité géographique des Facultés de Pédagogie parce que ne pouvant assumer les coûts d'une vie universitaire hors de leur région et loin de leur 
famille, ou encore ceux qui, au point de départ, sont dans un état de dénuement économique, social ou relationnel tel, qu'il leur faut d'emblée tabler sur les Facultés de Pédagogie, car c'est là que l'emploi est - ou était jusqu'ici - le plus garanti. Présomption de médiocrité qui pèse sur les enseignants, d'autant plus paradoxale qu'elle est à la mesure même de ce qu'a été cette gigantesque opération de modernisation de la société par l'école. Ainsi, et par un renversement des rôles, ceux qui se considéraient comme « victimes » d'un retrait de l'État qui prenait le sens d'une trahison, ou du moins d'un manquement à la promesse (d'embauche), ont été désignés par les discours autorisés comme coupables potentiels.

7 Examinons maintenant les justifications de ces derniers, candidats aux postes d'enseignants, car pour n'être pas une « foule », ce rassemblement n'en a pas moins son "économie morale" (Thompson, 1971, p. 76-136). Le rassemblement n'est pas homogène et cela nourrit en partie les arguments, chacun s'estimant plus sujet à l'injustice que d'autres. Premier clivage, le concours n'était pas ouvert qu'aux seuls diplômés des Facultés de Pédagogie, mais à ceux des autres facultés également ; à quoi servent alors les Facultés de Pédagogie? Question endémique qui revient épisodiquement dès le début des années 1930, moment de la mise en place du premier Institut de pédagogie. Héritant des anciennes écoles normales, celui-ci tente de consolider sa mainmise sur la production de la profession enseignante. Il ne peut qu'entrer en conflit avec l'Université d'État (1925), guère plus vieille. Les diplômés en histoire de la Faculté des Lettres, ou en mathématiques de la Faculté des Sciences, que pouvaient-ils faire d'autre sinon enseigner? Fortement présente au sein du ministère de l'Instruction publique, la corporation pédagogique, dont Ismâĉl al-Qabbânî (1898-1973), ministre entre 1952 et 1954, reste la figure emblématique, avait imposé le passage par l'Institut de Pédagogie à la suite des études universitaires, comme condition d'accès aux postes d'enseignants. Au grand dam des universitaires, bien entendu. C'est ainsi d'ailleurs, et en attendant que se creuse de manière irréversible la distance entre enseignants des universités et des écoles, que parmi les figures fondatrices des sciences humaines (histoire, géographie) et naturelles, il en est un bon nombre qui rédigent leur thèse de doctorat et accèdent aux premiers signes de la reconnaissance académique qu'ils contribuent à créer, tout en enseignant dans les écoles, ou en travaillant dans l'inspection scolaire, une situation difficilement pensable aujourd'hui'. Jusque dans les années cinquante, la compétition est vive et l'observation des parcours de diplômés montre que les va-et-vient entre pédagogie et sciences humaines sont encore possibles. Le lancement d'une politique de scolarisation à grande échelle dans les années 1950 casse le monopole pédagogique sur le métier d'enseignant, en réduisant à des "stages" l'exigence de formation pédagogique des diplômés des universités, rendant ainsi caduque la vieille compétition. C'est qu'alors l'État avait besoin d'enseignants, mais l'image de la profession ne s'en est pas mieux portée et durant ces années de "transition socialiste » la pédagogie et ses formations sont taxées d'américanophilie par certains intellectuels pour qui « l'enseignant pédagogue » est la figure même du bureaucrate et tout l'inverse de ce que devrait être l'enseignant, « philosophe dans la cité » ('Awad, 1964, p. 156).

8 Les années 1990 voient ressurgir la question du primat du pédagogique : rareté des postes et pléthore de diplômés, mais plus fondamentalement une interrogation sur l'existence même des Facultés de Pédagogie, incriminées pour la faiblesse de la formation ou parce que les islamistes y seraient plus nombreux qu'ailleurs. Un diplômé 
en histoire serait-il plus apte à l'enseignement que son collègue formé à «l'enseignement de l'histoire »? C'est ainsi et en mettant en avant la spécificité de leur double compétence, que les candidats formés à la pédagogie s'estiment doublement lésés : et par le principe même du concours et par le fait qu'il soit ouvert à d'autres candidatures.

Dans le rassemblement, il faut compter avec les candidatures rejetées d'emblée parce que non conformes : le concours n'était ouvert qu'aux diplômés de 1998 et 1997. C'était au tour des diplômés des promotions antérieures d'entonner le couplet sur «la méconnaissance par les responsables politiques des réalités de la condition du peuple ». Non, le temps à lui seul ne suffit pas à régler le problème du chômage comme font semblant de le croire les responsables et nombre de diplômés des promotions antérieures déclarent ne pas avoir trouvé d'emploi. Ils demandent si le fait d'avoir un «boulot " précaire plus ou moins rémunérateur mais peu considéré pour un diplômé de l'université - chauffeur de taxi, coursier, serveur, vendeuse - implique un renoncement à la dignité (relative) d'un poste d'enseignant ? Sont mis en avant les avantages de la fonction publique : non point le salaire, mais la stabilité, la couverture sociale et une fiabilité de l'État-patron que n'a pas le privé.

Ce même rassemblement fait, enfin, l'objet d'un " procès en discrimination ». C'est ainsi du moins que des diplômés de l'université d'al-Azhar, chercheurs d'emplois dans l'enseignement, mais ailleurs que dans la filière scolaire azhari, stigmatisent un concours ouvert selon les termes malheureux du ministre aux diplômés de «nos universités ». Ils y voient un déni et de leur savoir et de leur appartenance à une nation qui n'a de cesse de louer "ses fils diplômés". Serionsnous les fils illégitimes s'interrogent en substance les diplômés d'al-Azhar? renouant là aussi avec l'interrogation sur le statut politique et savant de cette université, on ne peut plus étatisée et qui pourtant est systématiquement comptée à part.

11 Ultime paradoxe enfin celui de l'État tout à la fois recherché et tenu en suspicion ; alors même que les responsables s'étaient assuré la neutralité d'un ordinateur pour gérer le recrutement de manière infaillible, tout ce monde dénonçait un «népotisme informatique » qui en avait favorisé certains.

Le rassemblement s'est dispersé, laissant posées autant d'interrogations sur les destins individuels qu'il comptait d'individus. Embauche ou concours? La question elle, est revenue à plusieurs reprises et de deux manières au moins.

L'une a pu être qualifiée de "violente ». Ce sont, reprenant un schéma qu'on retrouvera plus loin, les étudiants en pédagogie qui «s'inquiètent pour leur avenir ». En avril 1999, grèves et manifestations essaiment à partir de la plus ancienne des Facultés de Pédagogie, située en dehors du campus au plus loin des traditions prestigieuses du mouvement étudiant et de ses formes d'action collective. Plus proche dans les représentations d'une école secondaire prolongée et cela n'est pas étranger à la stigmatisation de la grève des étudiants ou à l'admiration surprise qu'elle suscite chez d'autres. Manipulation d'une masse par une poignée d'éléments politisés ou revendications illégitimes parce qu'inhabituelles venant de la part d'étudiants qui se mobilisent en principe pour des «causes " (l'Iraq, la Palestine, la Démocratie) et non des « raisons » et doivent rester au-delà de l'expression des intérêts privés. La grève est en tout cas durcie puis cassée par l'intervention massive des forces de l'ordre et les arrestations d'une vingtaine de "meneurs " - et de meneuses faudrait-il rajouter puisqu'on compte nombre d'étudiantes. "Ayantsdroit» spoliés, c'est à ce titre que 
s'expriment les étudiants en pédagogie, mettant en avant la « rupture du contrat » sur la base duquel ils avaient "choisi » les Facultés de Pédagogie: l'embauche systématique. La grève a, elle aussi, pris fin, mais point la question.

Elle revient épisodiquement sur le mode médiatique. Dernier épisode en date, la «vidéoconférence» du ministre de l'Éducation en mai 1999. Cela se voulait consultation nationale, plus ou moins fermée, des représentants des diverses parties prenantes de l'éducation nationale (élèves, parents d'élèves compris) et on peut imaginer les tris politico-administratifs préalables à l'organisation de l'événement. Cela aurait pu ne pas en être un si la presse n'avait été conviée à couvrir et commenter la vidéoconférence et à rendre compte de débats ultérieurs autour des thèmes examinés. Ainsi mis en scène, dans un cadre unificateur et national, le consensus pouvait souffrir l'expression des dissensions et doléances. À la question récurrente, la réponse du ministre est en substance: "de l'embauche systématique il n'y en aura point; des postes, il y en aura pour tout le monde ». Réponse illogique en apparence, mais qui ne l'est peut-être pas tellement au regard d'une stratégie politique (et pédagogique) qui consiste à détromper sinon casser des attentes admises comme légitimes, tout en rassurant. À croire que l'État demande à ses citoyens chercheurs d'emplois de faire l'apprentissage de l'aléa qu'il ne peut prendre à son compte que dans une mesure restreinte.

Quoi qu'il en soit, la récurrence de la question - et qu'il s'agisse d'éducation nationale n'est pas anodin - signifie bien que, rupture d'un contrat ou non, les parties prenantes de ce débat ont encore des choses à se dire. On ne peut savoir pour combien de temps, mais ces choses-là ne datent pas d'hier.

Années 1930 : le chômage des diplômés construction d'une question politique « On ne trouve pas en Égypte jusque vers les années trente au moins, de phénomène comparable à cette fraction de la classe des intellectuels russes du XIXe siècle qui, ne s'intégrant pas ou s'intégrant peu à la société et à l'État, tendit à se constituer comme un groupe en marge, avec ses idées et ses valeurs propres. C'est que longtemps, en Égypte, l'intégration s'est effectuée sans trop de difficultés. D'abord les jeunes diplômés trouvent des postes de travail; ensuite, il y a en gros communauté de valeurs entre les jeunes et les moins jeunes et l'État et la société civile (appelons ainsi la part de la société qui parvient à s'exprimer). Les étudiants et aussi les élèves des écoles secondaires, fournissent des militants et des manifestants et participent aux luttes politiques conduites par les aînés (..) Il est presque toujours question en fait, de réformer l'État, d'améliorer son rendement social, rarement de changer les structures de la société et de l'État " (Delanoue, 1985, p. 19-30).

De ce propos qu'avance Gilbert Delanoue, retenons la «communauté de valeurs". Retenons également ces accès en chaîne à l'éducation, à la bureaucratie et à l'élite politique ; ce sont de tels accès qui fondent tout à la fois l'appareil d'État moderne, le champ politique et l'autodéfinition des intellectuels (Alleaume, 1987/1988, p. 67-87). Ces éléments, il faut les avoir présents à l'esprit en abordant le moment où est construite, dans les années 1930, la question du chômage des diplômés. Dire qu'une question est "construite» n'est pas suggérer qu'elle soit artificielle ou dénuée de fondements. La déclaration du ministre des Finances d'alors est bien réelle, qui estime à 11000 les diplômés auxquels on n'avait pu donner d'emplois. Ce qui n'interdit pas - on y reviendra - de questionner la logique du comptage, tout à la fois la liste et le «moment » où elle est établie. Outre les effets locaux de la "grande crise » des années 1930, limitant la création d'emplois, vers le milieu des années 1920, le nombre des 
inscrits dans certains établissements d'enseignement supérieur avait augmenté et se traduisait maintenant par une augmentation des diplômés chercheurs d'emploi. Réelle également - sinon plus - "la souffrance des diplômés et de leur famille " selon les termes d'un observateur (Matar, 1939, p. 6).

Ce n'est donc pas le caractère « objectif » de la question du chômage des diplômés qui est en cause ; ce à quoi on s'intéressera est son « mode d'objectivation »; autrement dit, les formulations d'une question sur le chômage des diplômés, analyseur de leur potentiel et du poids moral et politique d'un pacte non écrit.

Pour introduire à cela, on s'arrête sur l'inflation discursive qui caractérise le moment. Sa source n'est pas difficile à identifier et la bibliographie sur la question n'aurait sans doute pas été la même si elle n'avait été initiée par le "concours de journalisme ». En 1936 et durant les 100 jours que dure son gouvernement, le Premier ministre cAlî Mâhir, lance un concours de journalisme. Des prix sont proposés aux meilleurs écrits sur les thèmes suivants : al-Azhar; langue, religion et coutumes; la motivation personnelle pour la réforme sociale; le chômage et le rôle de la spécialisation régionale de l'enseignement dans son éradication; l'éducation patriotique indépendante; les moyens $\mathrm{du}$ succès pour l'homme du $\mathrm{XX}^{\mathrm{e}}$ siècle; le renforcement de la vie constitutionnelle et de l'unité nationale et le patriote éclairé; le progrès social du paysan ; la renaissance de la femme ; l'hymne national.

19 Au moment de la remise des prix, c'est un gouvernement wafdiste qui est au pouvoir, mais l'héritage textuel est resté. Le moment - l'Égypte vient de signer l'accord de 1936 contesté par certains et qui, pour d'autres, inaugure une nouvelle phase de son indépendance politique - justifie largement de l'intérêt pour ces questions : comme au lendemain de la déclaration britannique de 1922, après l'achèvement de ce qui est considéré comme une avancée significative de l'indépendance, vient pour les élites, le temps des réformes internes. Il est troublant de constater que la bibliographie de l'époque est, pour une large part, suscitée par un « appel d'offre ", des écrivains étant sollicités par des pouvoirs publics en quête de « solutions » aux " problèmes sociaux ». Pour la question qui nous concerne en tout cas, c'est de là que procèdent, pour une bonne part, les écrits sur le chômage, primés ou non, ouvrages ou articles allant de la revue de vulgarisation scientifique Al-Muqtataf à l'Égypte contemporaine éditée par la Société royale d'économie politique. Avec un ministère des Affaires sociales (1939), sa revue, et les écrits de ses fonctionnaires, c'est un peu la même scène qui se répète bien qu'elle rende compte de l'accumulation de nouveaux savoirs sociaux, liés à la constitution d'administrations pour le «placement» des diplômés, ce qui n'est pas exactement la gestion du chômage. Toujours est-il qu'au travers de ces énonciations, l'enjeu semble être le même : la désignation consensuelle et la mise en forme de l'objet chômage, au même titre que la femme, le paysan et la Constitution, l'invention d'un espace de débat et l'unification des termes de celui-ci. Non pas tant exutoire que définition de ce que serait une parole légitime et signifiante, sens et légitimité étant une seule et même chose.

Dans l'Égypte monarchique et libérale, le vocable " question politique » était réservé à l'indépendance nationale. Question sociale englobait le « reste », dont l'heure viendrait une fois l'accès à l'indépendance réalisé. Avec le « chômage des diplômés » posé comme question politique, c'est peut-être la première fois que l'ordre politique s'énonce et se pense en tant que tel : moins sous l'effet d'une " menace » externe d'ailleurs que pour des impératifs de cohérence interne, car c'est de l'intérieur même de cet ordre que se 
pose la question. Touchant les diplômés, cette question est au cœur des dynamiques de production de l'élite sociale et politique; elle ne s'inscrit pas dans le spectre des préoccupations «sociales", qui, elles, concernent des «classes dangereuses » dont il faudrait adoucir le sort et les mœurs.

21 Significativement, en relisant les comptes rendus de cette situation de "crise", l'observateur ne peut faire la part entre consensus et contestation. Ce sont des mêmes acteurs que procèdent des prises de parole différentes par la forme, identiques sur le fond. Par ailleurs, il faut relever l'antériorité d'un débat (et d'un vocabulaire) sur le chômage ouvrier, question sociale par excellence, et on aurait pu s'attendre à ce que cette "technologie sociale » soit mobilisée; or, on ne fait appel à celle-ci que pour mieux le rejeter, car ces deux chômages là sont différents de par leur " nature ». Il faut prendre au sérieux les interrogations que suscite le chômage des diplômés et qui traversent trois bilans, indissociables mais combien difficiles à concilier pour les acteurs, sur un plan tout à la fois logique et moral: questionner l'école, le fonctionnariat et ce que serait une "économie nationale» est pour l'époque, questionner les fondements d'un ordre.

L'histoire politique a déjà traité des accompagnements politiques du chômage des diplômés : manifestations, cortèges et grèves ont tous leurs « dessous » politiques. Car « les années de la jeunesse » (1935/1936), c'est également le moment d'une mobilisation nationaliste et d'une visibilité politique toute nouvelle de la jeunesse étudiante, prise dans des rapports de conflit / cooptation, avec les aînés de l'establishment politique. Lesquels jouent les "apprentis sorciers" selon l'expression de Jacques Berque, en manipulant une charge - les chemises de couleur, milices de parade constituées par les partis politiques rivaux - dont ils ignorent le potentiel explosif (Berque, 1967, p. 477). Que les aînés «courtisent » les jeunes étudiants, cela est vraisemblable; au-delà des raisons instrumentales et de l'attention portée aux conflits - radicalisation et contestation de l'ordre - il semble possible d'identifier également de profondes connivences - sinon une "communauté des valeurs" comme on l'a dit plus haut entre les jeunes diplômés et leurs aînés.

Elles apparaissent au travers de mobilisations moins visibles ou plus intellectualisées. C'est au bénéfice d'une lecture généreuse qu'on peut traiter de "professionnels" certains cercles, amicales ou corporations qui auront leur mot à dire sur le chômage des diplômés. Ce à quoi on a affaire le plus souvent, est un regroupement sur la base, non des conditions d'exercice de la profession, mais de l'établissement de formation d'origine : s'y retrouvent diplômés, étudiants et enseignants. La diversité des âges, des statuts et des fortunes professionnelles est « rattrapée » par un rapport paternaliste entre jeunes et aînés et ce qu'il implique en termes d'obligations réciproques. C'est ainsi que s'explique par exemple, la Note sur les demandes des étudiants et diplômés de la Faculté de Commerce avec annexe sur les postes que pourrait occuper la promotion 1937, présentée par le Club du Commerce, présidé par un pacha et placé sous le patronage royal. On en retient une formulation non antagoniste des intérêts entre diplômés en fonction, au chômage et étudiants, qui se consolide à l'encontre des nouveaux arrivants. En amont, ils réclament une politique malthusienne pour ce qui est de l'accès à la Faculté de Commerce, y compris par la hausse des droits d'inscription; en aval, des postes réservés et l'exclusion des incompétents en faveur des qualifiés. Autre exemple, le congrès de l'Association des diplômés de l'École normale supérieure, durant lequel dirigeants de l'association, enseignants mais aussi responsables politiques, ministre des 
Finances en tête, ont débattu pendant trois jours du chômage (juin 1934). Rajoutons que pour les enseignants, c'est le moment où, en marge mais en lien avec le chômage, s'expriment, de manière autonome et en rupture avec les collectifs, certaines insatisfactions; les "mal-embauchés» des écoles libres revendiquent les mêmes avantages que les enseignants fonctionnarisés. On retrouve là, sans jonction aucune entre les établissements de formation, et alors même qu'il s'agit des mêmes conditions d'exercice du métier, deux "cahiers de doléances» identiques; l'un émane des enseignants des Écoles libres issus de l'École normale, et l'autre, des enseignants des écoles libres issus l'École de langue arabe (Groupe École normale supérieure, 1936, Groupe École de langue arabe, 1936).

Écrits savants faisant le détour par Alfred Sauvy ou brûlots d'al-Shabâb, porte-parole de tout ce que l'Égypte compte de "chemises de couleur", ont pu se rencontrer. L'exemple en serait Le Jubilé d'argent (1936) qui retrace 25 ans de la vie de l'École (1911), puis École supérieure (1921) et enfin Faculté de Commerce (1935). Il n'est pas de trace plus parlante de la « communauté de valeurs » mentionnée plus haut. Le Jubilé d'argent ne ressemble en rien aux publications commémoratives et loin de l'attendrissante photo de groupe à l'adresse des générations futures et pour laquelle on fait généralement "bonne figure ", c'est un instantané de la crise. Y figure notamment la liste des diplômés et les postes occupés depuis la fondation de l'établissement, avec des blancs de plus en plus nombreux pour les dernières promotions. Les étudiants ont mené des interviews avec les ministres de l'Instruction, de la Justice, et avec Talcat Harb, fondateur du groupe Misr, premier groupe national industriel et financier. $\mathrm{Au}-$ delà des réponses à des questions qui les comportent déjà, Le Jubilé d'argent commémoration dans la commémoration - relate dans le menu détail les festivités de celui-ci. Cela avait commencé par un déjeuner et s'était achevé tard dans la nuit à l'Opéra du Caire, avec un récital d'Umm Kulthûm. Entre temps, tout ce que l'Égypte compte de sommités s'était retrouvé pour le thé, les jeux et concours sportifs, et les activités des étudiants. En 1934, la troupe de théâtre de la Faculté avait déjà présenté Le chômage. Là il s'agissait du Fonctionnaire. C'est l'histoire de deux diplômés : l'un, riche héritier, se contente d'un poste de fonctionnaire, alors que l'autre, pauvre, reprend et fait fructifier le modeste commerce de son père. Or voilà que les deux hommes sont amoureux d'une jeune fille: l'aventureux est éconduit, le fonctionnaire prestigieux retenu, mais guère longtemps. Accusé de corruption, il perd son poste et ne trouve à s'employer qu'auprès de son rival d'antan et pour un salaire plus élevé.

La morale de l'histoire a dû recueillir l'assentiment de ceux qui étaient là durant la soirée. Mais ce n'est qu'une partie du message qui parcourt l'ouvrage commémoratif ; le self-made man est un choix qui n'est pas donné à tous et si ce n'est un tromperie, c'est une chimère. Les deux propos émanent d'une seule instance et il est bien difficile de classer Le Jubilé d'argent - imprimé par les soins de l'imprimerie du Groupe Misr, dont on attend qu'il soit pourvoyeur d'emplois - comme publication officielle ou comme l'expression d'une contestation.

Entre revendications et diagnostics, entre contestataires, analystes et décideurs, la distance sociale est moindre que celle qui, pour d'autres groupes, sépare la masse perçue comme aphasique et silencieuse - de ceux qui s'en font les porte-parole. Là, contrairement aux ouvriers "qui ne peuvent se représenter eux-mêmes ", ce sont ceux qui parlent qui produisent les représentations : non seulement les diplômés parlent 
pour eux-mêmes, mais tout parle pour eux. On le voit à la manière dont la généalogie ouvrière du chômage est niée.

Dans ces mêmes années, "travail ouvrier » est une redondance. 'Amal (travail) semble désigner exclusivement l'activité des cummâl (ouvriers). Face à " travail et travailleurs ", et d'autant plus cohérente, n'existe que la wazîfa (fonction ou poste) des diplômés et les réglementations de leur emploi dans la fonction publique. Dans ces mêmes années également, l'acception du chômage comme absence de travail est devenue évidente. Batâla (chômage) n'est plus l'oisiveté des rentiers ou la paresse des pauvres, même si les vieilles acceptions et leurs contenus moraux sont loin d'avoir disparu. Vers 1930 enfin, et pour couper court notamment à l'élaboration d'un code du travail unifié, un Bureau du travail relevant du ministère de l'Intérieur est mis en place, chargé tout à la fois d'arbitrer et de "surveiller " les conflits de travail et de mener des études sur diverses questions, dont le chômage. Sur celui-ci, comme sur l'ensemble de la condition ouvrière, la mission de Harold Butler, expert de l'office international du travail de 1932, est décisive. On pourrait en résumer les conclusions ainsi: à industrie embryonnaire, lois à la mesure ; les catégories juridiques sur-construites auraient des effets de retour sur les individus et un arsenal de lois entraînerait un surcroît de revendications infondées. Le chômage ouvrier en Égypte est faible par rapport à l'Europe et, habitués à l'aléa et à vivre de peu, les ouvriers égyptiens ont des besoins infimes par rapport à leurs collègues européens. Ni code unifié ni législation contre le chômage donc, ni bourse du travail, « ce serait trop tôt ", ni assurances car elles sont prématurées, et qu'il faudrait pour cela comptabiliser les chômeurs; opération que rend difficile le travail intermittent qui empêche de cerner avec précision les ouvriers durablement chômeurs. En 1931, l'échec d'un comptage - 24000 chômeurs «même multiplié par deux c'est peu par rapport à l'Europe » dira Butler - confirmerait pour la suite que les ouvriers ne "peuvent se classer eux-mêmes ». Et quand bien même on parviendrait à les répertorier pour leur verser des assurances, n'est-ce pas une injustice flagrante vis-à-vis de milliers de travailleurs agricoles? (Butler, 1965, p. 145-170). Ne reste alors que les bureaux de placement. Relevant de la tutelle du ministère de l'Intérieur, puis des Finances et enfin des Affaires sociales, les bureaux avaient été pensés à partir des questions ouvrières, mais sont mis en place pour les «diplômés chômeurs » en 1936 et n'acceptent les demandes des ouvriers qu'en 1937. Est-ce à dire que les deux chômages relèvent d'un même traitement? Ou encore qu'il s'agit d'un même " travail »? Rien n'est moins sûr et la « lutte contre le chômage " n'acquiert ses titres de noblesse (encore sont-ils modestes) qu'à partir du moment où elle concerne les diplômés.

Aussi dérisoire qu'il soit, un espace de débat existe autour de la question ouvrière et du chômage ouvrier, lieu de réflexion, d'importation et de production de savoirs sociaux. À ces antécédents, les protagonistes du débat ne font pourtant pas appel et c'est le sociologue 'Alî ‘Abd al-Wâhid Wâfî, deuxième lauréat du concours mentionné plus haut, qui pose d'emblée la chose : «il y a deux chômages » (Wâfî, 1936, p. 16). Sur ce fait, experts et hommes de plume sont d'accord; chômage ouvrier - «celui dont souffre l'Europe » - et chômage des diplômés - «celui auquel nous faisons face » - sont deux questions différentes. C'est ce qui autorise à dire que l'un relève en effet de la "question sociale » alors que l'autre ressortit pleinement d'une " question politique ». Dans le débat sur le chômage des diplômés, il n'a jamais été question d'assurance car le 
diplômé ne peut être associé à l'indigence. D'ailleurs, d'assurance, il n'en réclame point et ayant son " sens de l'honneur ", ce qu'il cherche c'est un emploi.

La sophistication du débat sur le chômage ouvrier est visible dans l'introduction d'une variable supplémentaire, "la durée du chômage» (Topalov, 1994); c'est là qu'intervient le moment où est établie la liste. Depuis le début du siècle existe une statistique scolaire rassemblée par les soins du ministère des Finances et qui comporte un état des diplômés ; ils poursuivent des études à l'étranger ou en Égypte, sont sans occupation, ont des emplois ou leur statut est inconnu. Ces données sont recensées en juin et publiées en décembre de la même année. Accumulation de données et mesure de l'État, il s'agit également de flux d'informations qui renseignent sur l'existence de liens sociaux en même temps qu'ils les construisent. En ce sens, les séries sont aussi « texte " et en relisant celui-ci, on peut imaginer qu'au début du $\mathrm{XX}^{\mathrm{e}}$ siècle, bon nombre de diplômés « sans occupation » s'apparentaient à des rentiers oisifs; or, vers le milieu des années 1930, ils étaient tous assimilés à des « demandeurs d'emploi» (dans nos termes contemporains). On est loin du temps où des rapports personnalisés traitaient dans le menu détail tout ce qui concernait la vie des Égyptiens envoyés en mission à l'étranger, loin du temps où un Hifnî Nâsif (1855-1919)' prenait ses fonctions de maitre au lendemain de l'obtention du diplôme de l'École de langue arabe (1882). Il est vrai qu'alors, la promotion était constituée de deux élèves. Les établissements d'enseignement s'investissant dans la promotion de leurs diplômés, on peut comprendre en quoi l'information est «lien» et du coup la réaction du doyen de la Faculté de Commerce à une question d'al-Shabâb: "Comment peuton recenser nos diplômés chômeurs s'ils ne répondent pas aux questions ?! Après tout ils ne sont peutêtre pas très nombreux » (al-Shabâb, 27 avril 1936). Dans cette même livraison, la revue publie un décompte de l'état des diplômés de la Faculté de Commerce entre 1920 et 1935 ; sur l'ensemble de la période, les 100 chômeurs et non-réponses, qu'on ne peut distinguer, sont concentrés sur les diplômés de l'année en cours ; sur ces derniers, qui sont au nombre de 119, 58 seulement avaient un emploi quelques mois après l'obtention du diplôme alors que 61 d'entre eux n'en avaient point. On peut ainsi constater que, concernant les diplômés, «durée du chômage », cette notion centrale dans la construction d'une " condition ouvrière », est une catégorie qui non seulement est inopérante mais relève du non-sens. À aucun moment, ce débat ne spécifie la durée, sinon l'attente, au-delà de laquelle un diplômé est bel et bien "légitimement" chômeur.

Par ailleurs, il est ou n'est pas chômeur de manière catégorique et intégralement. Le travail intermittent du diplômé n'est pas une catégorie mobilisée; pas plus d'ailleurs que la distinction - demeurée théorique il est vrai - entre under-employed et unemployed, valable pour les ouvriers. Selon celle-ci, il convenait non pas de classer la main-d'œuvre (cela s'avère impossible), mais de classer les causes du chômage elles-mêmes (Hamdî, 1944, p. 20). S'agissant de diplômés, les trois pistes explorées par les observateurs carences du système d'enseignement, grandeur et misère du fonctionnariat et absence d'un secteur national productif - cumulent plus qu'elles ne classent les causes du manque d'emplois et celles renvoyant à la mauvaise répartition de ceux qui existeraient.

31 L'inventaire des différences entre les deux chômages va plus loin; l'industrie étant jeune et en phase d'expansion, le sort de l'ouvrier ne peut que s'améliorer et son chômage n'est qu'une question d'ajustement entre cycles. Que dire d'un diplômé dont 
le principal employeur est un appareil d'État «parvenu à saturation»? De plus, alors même que l'ouvrier peut monnayer sa force de travail et se reconvertir, que dire d'un diplômé qui ne sait faire que ce pour quoi il a été formé ? On n'a pas à se poser à son propos ces questions qui hantent les nuits des réformateurs, au sujet des cireurs de chaussures ou des vendeurs de billets de loterie. Ces derniers sont de faux travailleurs, migrants indésirables dans la ville, et pour les classer, on hésite non entre chômage et travail, mais plutôt entre vagabondage et criminalité. On croit savoir, d'ailleurs, quels désordres sociaux peut engendrer leur misère, alors que ce qui guette le diplômé au chômage, c'est le déclassement et non la loi sur le vagabondage. Quelles que soient les divergences sur l'ampleur du chômage des diplômés, tous les protagonistes de ce débat, se retrouveraient dans le constat suivant : «même peu, c'est beaucoup " (Association des diplômés de l'École normale supérieure, 1934, p. 214). C'est cela la mesure " objective » du phénomène et on a des raisons de croire que la manière de rendre compte du phénomène et de le rendre visible, intervient dans sa massification et non simplement l'inverse.

32 C'est ce qui fonde l'écart durable entre un droit $d u$ travail - inscrit dans l'ordre des préoccupations sociales et lié à la condition ouvrière - et un droit au travail des diplômés, jamais codifié et pourtant fondateur d'attentes unanimement reconnues comme légitimes et qui sont à la base de l'ordre politique.

«Droit au travail, jamais codifié »? Il y a de quoi s'interroger sur la pertinence de cette formule. Pour revenir à un point de détail évoqué plus haut, on avancera que, tout comme la question du placement a esquivé celle de l'assurance-chômage pour ce qui est de la condition ouvrière, ce à quoi on a affaire pour les diplômés est bien une embauche par l'État qui pour un temps aura paradoxalement garanti l'emploi, tout en esquivant le droit. Au-delà des changements de régimes politiques, c'est ce que semblent confirmer également les lois des années 1960 relatives à l'embauche des diplômés.

Comparer : le droit et la loi

34 Entre l'Égypte « libérale » des années 1930 et l'Égypte « néo-libérale » des années 1990, les analogies peuvent être hasardeuses. Les deux situations abordées plus haut l'ont été de manières différentes. Les traces sur lesquelles on s'est basé le sont également. En outre, s'agissant des diplômés au chômage, privilégier l'échelle ou retenir la proportion donnerait à voir des choses différentes. La massification évidente des années 1990 ne devrait pas faire oublier l'ampleur des « effets idéels » de la situation qu'avaient créée 11000 chômeurs dans les années 1930. Enfin, dans l'entre-deux que représente l'Égypte nassérienne et sur lequel on reviendra, l'embauche des diplômés par l'État a eu des formalisations inscrites dans des visées différentes; l'économie nationale planifiée, dans laquelle le secteur public prenait le rôle moteur, et l'élargissement de l'appareil d'État à la mesure de ce qu'il fallait assurer en termes de services / droits (santé, éducation) garants de justice sociale, s'inscrivaient dans ce qu'on aurait voulu être de nouveaux rapports entre "les masses» et des dirigeants qui disaient n'avoir de légitimité que parce qu'ils en émanaient. Le manquement à la promesse d'embauche prenait d'autant plus le sens d'un abandon. On s'en tiendra donc à quelques remarques purement phénoménologiques qui font d'autant mieux ressortir les paradoxes de l'entre-deux.

35 À commencer par les péripéties du placement. Dans les années 1940, la Deuxième Guerre mondiale avait permis au bureau créé à cet effet de placer diplômés et ouvriers au service de l'effort de guerre de l'armée britannique. S'agissant du privé, le 
gouvernement empruntait un nouveau langage aussi sibyllin que menaçant à l'égard des employeurs potentiels : « en dépit des nombreuses propositions qui visent à mettre fin au chômage des diplômés par les moyens de la législation, le gouvernement préfère compter sur le soutien volontaire des hommes d'affaire » (Hamdî, 1944, p. 368). Dans les années 1990, la justification socioéconomique des entrepreneurs tient à leur capacité présumée à générer des emplois (et des devises). Avec l'achèvement de l'embauche systématique des diplômés, outre la mise à l'honneur du concours dont on a vu plus haut un exemple, c'est aussi à la « réinvention » du placement qu'on assiste. Outre les canaux informels et les petites annonces, relais habituel des offres d'emploi, un nouveau Bulletin national pour l'emploi centralise les offres peu compétitives de même qu'a été évoquée la mise en place d'une Agence nationale pour l'emploi sur le mode de l'ANPE (française).

Les établissements d'enseignement supérieur se sont investis d'une responsabilité publique ou privée ? - dans la quête d'emplois. Dans les années 1990, cela prend la forme de "journées pour l'emploi » qu'organisent les Facultés pour la promotion de leurs diplômés et où sont conviés les employeurs éventuels. Dans les années 1930, certains annuaires - mémoire par excellence des institutions éducatives - se donnaient cette mission exceptionnelle : Le Jubilé d'argent qu'on a vu plus haut en est un exemple. D'autres démarchaient auprès des employeurs éventuels ou consacraient une publication à ce seul effet (École intermédiaire de commerce, 1938). D’autres encore reprenaient à leur propre échelle la comptabilisation des diplômés et le suivi de leur carrière. Produisant son propre recensement au début des années 1950, la Faculté des Lettres pouvait ainsi s'enorgueillir d'avoir " pisté » 1500 de ses 1972 diplômés (dont 33 décédés); sur les 473 «manquant à l'appel» il faut compter les ex-étudiantes converties en femmes au foyer, ceux qui poursuivent des études à l'étranger et les nonréponses (Faculté des Lettres, 1951). Dans leur écrasante majorité, les diplômés étaient fonctionnaires, enseignants ou enseignants fonctionnarisés.

Dans les deux situations examinées sont construites des figures de la négativité sociale, signes de l'altération de dispositifs bons en eux-mêmes. "L'effet pervers " est à rechercher dans les figures du bureaucrate corrompu et improductif ou du «semiinstruit », qui brouille des frontières qu'on aurait voulu bien tranchées. En parallèle, le self made man, "son propre patron ", apparaît comme une figure positive. La figure de l'illettré, symptôme d'un manque, à laquelle on fait appel dans d'autres situations, brille par son absence. Dans une société où l'intégration se fait par l'école et où cette dernière est loin d'avoir touché l'ensemble de la population, c'est aux plus éduqués des éduqués qu'est reconnue la compétence à faire sujet politique. Les "non-éduqués » seraient-ils eux aussi, à l'instar du souverain dont Michel Foucault décrivait la présence invisible, "ce lieu parfaitement inaccessible puisqu'il est extérieur au tableau, mais prescrit par toutes les lignes de sa composition »? (Foucault, 1966, p. 29)

Dans les deux situations, au manque de travail ou encore à sa mauvaise répartition, répond l'argument d'incompétence des demandeurs d'emplois. Ainsi, si dans les années 1930, un nationalisme économique fustige la discrimination qu'exercent les entrepreneurs étrangers contre les diplômés égyptiens puisqu'ils préfèrent recruter dans leurs communautés, ce même discours pointe sur les faibles qualifications du diplômé produit par l'école égyptienne: ignorance des langues étrangères dans une économie aux mains des étrangers, mais de manière plus générale, ignorance des " règles du commerce social ", si on peut dire, des modes de présentation et de mise en 
valeur de soi et les commentaires d'époque décrivent tout en prenant sa défense, un diplômé fruste, peu civilisé, inapte à l'initiative et voué au destin de « rond de cuir » qui n'est plus assuré. Dans les années 1990, le propos est en substance l'inadéquation des savoirs reçus aux besoins d'un marché mondialisé et, en Égypte comme ailleurs, se développe une science du curriculum vitae: dans la qualification pour l'emploi, le diplôme stricto sensu n'est qu'un élément parmi d'autres, nécessaire mais pas suffisant.

Si les conduites revendicatives prennent des tournures différentes dans les deux situations examinées, l'emploi des diplômés reste au cœur de l'ordre politique et social. Parce qu'il y a une raison d'État qui ne correspond pas tout à fait à la logique des experts - commandités par ledit État - c'est ce dernier qui s'arroge la place du réformateur avec plus ou moins de douceur, renvoyant dos à dos les orthodoxies; toute expression des conflits et tensions est contrebalancée par la mise en scène et la réitération des consensus.

Cependant, dans les années 1990, il se dit, il est vrai, que la stabilité de l'emploi est inductrice de paresse, la grille de salaires unifiée contre-productive et le service public standard, rendu à des individus supposés équivalents, une aberration. Mieux, que toute gratuité - de l'enseignement ou de l'accès à l'emploi désormais conquête ou marchandise - est génératrice d'abus et, de ce fait, contraire au bien commun. À la mesure de l'État se conjugue désormais « l'État sur mesure » : small is beautifull pourraiton dire, et l'accroissement des rendements devrait passer par la réduction des effectifs. Toutes choses qui, dans l'Égypte des années 1960, étaient de l'ordre de l'indicible. C'est précisément à partir de considérations strictement inverses que l'État nassérien intervenait dans le monde du travail, de manière générale et sur la question de l'embauche systématique des diplômés en particulier. Il y a là aussi différentes traces allant de l'énonciation de l'obligation morale à la règle de droit.

41 Sur la première, le recul devrait sans doute permettre de repenser le statut de ces "grands textes» que les régimes socialistes avaient en leur temps promulgués; en l'espèce, la Charte nationale de 1960. Ce qui était alors "texte programmatique» apparaît a posteriori comme image idéalisée de ce que devrait être le lien politique et les obligations réciproques entre gouvernants et gouvernés (mais à cette image, il faudrait alors adjoindre les procès-verbaux des discussions qui ont présidé à la mise en forme de la Charte et montrent une fois de plus que le politique des années 1960 a été beaucoup moins monolithique que les analystes ne l'ont présenté).

42 Le travail est «droit, honneur et devoir », selon les termes de la Charte. Ce que reprend la Constitution (permanente de 1971, art. 13) en rajoutant : «il est garanti par l'État ». En Égypte, comme dans d'autres sociétés, se pose le dilemme de la reconnaissance théorique sinon rhétorique d'un droit au travail (et une obligation) énoncé, semble-t-il, en un moment où la «quantité de travail » n'était pas perçue comme un problème. En Égypte moins qu'ailleurs devrait-on dire de manière plus précise, puisque l'emploi des diplômés par l'État «semble" garanti dans les années 1960-1970 (et non comme ailleurs la matérialisation de ce droit en assurance-chômage).

Revenons pourtant sur la forme juridique de cette garantie. Qu'il s'agisse une fois de plus des «besoins de l'État» cela est certain; que ces besoins soient inscrits dans de nouvelles visées sociales et politiques, cela l'est aussi. Il serait trop facile, pourtant, d'établir une jonction directe qui ferait des normes juridiques la simple mise en application d'un droit. Un premier « décret présidentiel» (425 de 1962) porte sur la création dans les administrations de l'État, de postes pour les diplômés des Facultés 
dites théoriques restés sans emplois. En bénéficient également les fonctionnaires qui occupent des grades inférieurs à leurs qualifications. Un an plus tard, une deuxième loi (156 de 1963) reprend les mêmes dispositions, élargies à certaines catégories des diplômés des Instituts supérieurs, et supprime l'examen préalable à la prise de fonction, en vigueur jusqu'alors. En 1964, la loi 14 reprend les mêmes dispositions, valables pour deux ans; la formule est reconduite tous les deux ans avec des élargissements aux diplômés du technique. Ce n'est qu'en 1973 (loi 85) - année même où les investisseurs sont exemptés de certaines dispositions du droit du travail (loi 43) que les dispositions sont rendues permanentes. L'obligation d'État est abolie en 1986 et sans que l'une ou l'autre de ces lois ne fasse débat. À croire qu'aucun droit au travail n'avait été consigné.

Aussi longue que soit l'attente du tacyîn (nomination), et qui peut durer jusqu'à quatre ans car on embauche selon l'ancienneté des promotions, un diplômé des années 1960 et 1970 n'est pas un chômeur: il attend. Pour tromper l'attente, un service militaire conséquent et divers emplois provisoires d'appoint. Un amr takliff (obligation) guette ceux parmi les diplômés dont l'État a le plus besoin: enseignants, médecins ou ingénieurs. La nomination est dans ce cas obligatoire et équivaut à une période de service public rétribué. Chômage, ce vocable ne fait son apparition que lorsque l'attente devient de plus en plus incertaine.

Un premier paradoxe à relever est ce contraste évident entre le caractère largement provisoire des dispositions juridiques et l'ampleur et la stabilité des attentes et représentations. Un second oppose à ces dernières le silence qui entoure ces mutations pourtant capitales. Elles opèrent plus sur le mode du glissement que sur celui de la rupture. Il aura fallu que les mesures atteignent les candidats aux postes dans l'enseignement, pour que ressurgisse la question de la garantie d'emploi par l'État. Ce qui, bien sûr, ouvre sur la question des voies qu'a pu emprunter le travail des diplômés en Égypte ou en dehors de ses frontières (et question subsidiaire, une mesure de l'embauche «non-systématique » que l'État continue d'assurer par voie de concours).

Dans les années 1990, le retrait de la garantie d'emploi des diplômés n'augure pas forcément d'une réinvention pour le moins anachronique des «luttes» et conduites revendicatives ou technologies sociales - cela tient des deux - qui, ailleurs, ont été à la base d'un droit. Toutefois ce retrait ouvrirait sur des possibles: ce que serait par exemple le début d'une « histoire commune du salariat » couvrant ce spectre qui va des diplômés au dit "travail informel », et qui commencerait paradoxalement là ou certains prédisent la fin du travail (Méda, 1995). Cela n'équivaut pas exactement à une même gestion pour un unique chômage. "S'il nous faut vivre avec une armée de chômeurs, qu'ils soient au moins de ceux qui n'ont pas achevé leurs études» (Association des diplômés de l'École normale supérieure, 1934, p. 214). Ce propos des années 1930 est encore valable aujourd'hui et c'est là qu'opère le premier tri des "surnuméraires ", témoin semble-t-il d'un renouvellement à l'identique de ce qui reste au fondement du lien politique (Castel, 1995) : non pas tant le travail que l'éducation qui y donne accès et qui, elle même, n'est pas (encore) accessible à tous. Et toutes. 


\section{BIBLIOGRAPHIE}

cAwad L., 1964, Al-jâmica wa-l-mujtamac al-jadîd, (L'Université et la société nouvelle), Le Caire, Dirâsât ishtirâkiyya.

Al-cImari A., 1936, « La crise du chômage en Égypte et ailleurs, ses causes et ses remèdes », L'Égypte contemporaine, vol 24.

Alleaume G., 1987/1988, « La naissance du fonctionnaire », Peuples méditerranéens, 41-42, ÉgypteRecompositions.

Association des diplômés de l'École normale supérieure, 1934, « Congrès sur le chômage ", Alkitâb al-sanawî (Livre annuel), Le Caire, Imprimerie Al-ictimâd.

Berque J., 1967, L'Égypte, impérialisme et révolution, Paris, Gallimard.

Butler H., 1965, Nas taqrîr Butler (Rapport intégral de Harold Buttler), al-Talî‘a, juin.

Castel R., 1995, Les métamorphoses de la question sociale. Une chronique du salariat, Paris, Fayard.

Delanoue G., 1985, « Les intellectuels et l'État en Égypte aux XIX et XX`e siècles », Les intellectuels et le pouvoir, Dossiers du CEDEJ, Le Caire.

Faculté des Lettres, 1951, Al-Kitâb al-fiddi 1925-1950 (Livre d'argent), Le Caire.

Farag I., sept. 2001, « Les manuels d'histoire égyptiens. Genèse et imposition d'une norme », Genèses, $n^{\circ} 44$.

Foucault M., 1966, Les mots et les choses. Une archéologie des sciences humaines, Paris, Gallimard. Groupe Dâr al-culûm de l'enseignement libre, 1936, Muzakkira min jamấat Dâr al-culûm bi-l-taclìm alhurr (Note soumise par le Groupe de Dâr al-culûm enseignant dans les écoles libres), Le Caire.

Groupe École normale supérieure des écoles libres, 1936, Muzakkira bi sha'n islâh almadâris al-hurra wa tahsîn hâl al-mûdarrisîn biha (Note sur la réforme des écoles libres et l'amélioration des conditions des enseignants), Le Caire.

Hamdî H., 1944, Mushkilat al-batâla ; unemployment. Bahth cilmî wa dirâsa muqarana (Le problème du chômage, unemployment. Recherche scientifique et étude comparative), Le Caire, Jamấat al-kitâb.

Joseph I., 1996, « La compétence des rassemblements. Une ethnographie des lieux publics », Enquêtes, $\mathrm{n}^{\circ} 4$, La ville des sciences sociales.

Matar A. H., 1939, Al-taclîm wa-l-mutacatilûn (Enseignement et chômeurs), Alexandrie, Imprimerie de l'École industrielle.

Méda D., 1998, Le travail. Une valeur en voie de disparition, Paris, Flammarion, « Champs » (1 ere édition 1995).

Ministère de l'Instruction publique, École intermédiaire de commerce de Dâhir, mars 1938, Dalîl bi-asma' wa wazâ'if wa 'inwanât hadarât khirriji al-madrasa 1928-1937 (Guide des noms, fonctions et adresses de messieurs les diplômés de l'École depuis sa fondation), Le Caire.

Roussillon A., 1999, « Durkhémisme et réformisme. Fondation identitaire de la sociologie en Égypte », Annales, novembre-décembre.

Thompson E. P., 1971, «The Moral Economy of the English Crowd in the Eighteenth Century », Past and Present, vol 70. 
Topalov C., 1994, Naissance du chômeur 1880-1910, Paris, Albin Michel.

Wâfî A. W., 1936, Al-batâla wa wasâ'il al-jihâ wal-taclîm al-iqlîmî wa atharuhu fî cilâj albatâla (Le chômage, les moyens de son traitement et l'impact de la spécialisation régionale de l'instruction sur son éradication), Le Caire.

\section{NOTES}

1.Ils sont pour bon nombre d'entre eux issus de l'École normale supérieure. Ainsi du géographe cAwad, du scientifique Mucharrafa, de Kirdâni spécialiste en aéronautique ou des premiers historiens de profession (sur ces derniers Farag, 2001, p. 4-29).

2.C'est dire aussi combien était enviable le statut du fonctionnaire, outre le prestige de l'enseignement officiel, deux éléments qui contrastent avec la situation des années 1990.

3.Issu de l'École de langue arabe, où il assure un enseignement après son retour de mission en France, Wâfî est ensuite président du département de philosophie de l'Université. Il est l'introducteur de Durkheim en Égypte (Alain Roussillon, 1999, p. 1363-1394).

4.Dans l'ensemble de cette littérature, un seul auteur recommande « la création d'un fonds alimenté par les entrepreneurs, les subventions d'État et loteries. Et cela outre une revue et le versement d'allocations régulières aux chômeurs qui seraient attachés aux administrations et sociétés privées au titre de stages de formation en attendant les postes vacants ». (Imari, 1936, p. 455- 483).

5.Issu d'une famille aisée, proche dans les années 1880 des officiers insurgés dirigés par ‘Urâbî, Hifnî Nâsif a laissé une œuvre monumentale. Actif dans les cercles de lettrés, il s'intéresse à la rénovation de la langue arabe et passe pour l'un des humoristes de son temps. Père par ailleurs d'une progéniture nombreuse dont Malak, femme lettrée et poète, et Isâm, un des introducteurs de la pensée socialiste en Égypte.

6.La démonstration semble une reprise d'un ouvrage de Henry Beveridge (Christian Topalov, 1994, p. 364).

INDEX

Mots-clés : éducation, histoire, diplômés

\section{AUTEUR}

IMAN FARAG

Cedej 